"Synthesis, RNAi activity and nuclease-resistant properties of apolar carbohydrates siRNA conjugates" Vengut-Climent, E., Terrazas, M., Lucas, R., Arévalo-Ruiz, M., Eritja, R., Morales J.C. Bioorg. Med. Chem Lett., 23 , 4048-4051, (2013). PMID: 23764303, doi:

\title{
Synthesis, RNAi activity and nuclease-resistant properties of apolar carbohydrates siRNA conjugates
}

\author{
Empar Vengut-Climent ${ }^{\mathrm{a}}$, Montserrat Terrazas ${ }^{\mathrm{b}}$, Ricardo Lucas ${ }^{\mathrm{a}}$, Matilde Arévalo-Ruiz ${ }^{\mathrm{a}}$, \\ Ramón Eritja ${ }^{\mathrm{b}}$, Juan Carlos Morales ${ }^{\mathrm{a} *}$ \\ ${ }^{a}$ Department of Bioorganic Chemistry, Instituto de Investigaciones Químicas, CSIC-Universidad de \\ Sevilla, Americo Vespucio, 49, 41092, Sevilla, Spain \\ ${ }^{b}$ Instituto de Química Avanzada de Cataluña, IQAC, CSIC, CIBER-BBN Networking Centre on \\ Bioengineering, Biomaterials and Nanomedicine, Jordi Girona, 18-26, E-08034 Barcelona, Spain
}

ABSTRACT. Oligoribonucleotide conjugates carrying apolar carbohydrates at the $5^{\prime}$-end and the corresponding siRNA duplexes have been prepared using phosphoramidite chemistry. All the carbohydrate-siRNA derivatives were compatible with RNA interference machinery if transfected with oligofectamine. In the absence of a transfection agent, some of them exerted certain reduction of gene expression. Double-tailed permethylated glucose conjugated to siRNA through a long spacer inhibited gene expression up to $26 \%$ compared to the scrambled duplex. Such modifications contribute positively to the stability of oligoribonucleotides against 5 '-exonuclease degradation.

Oligonucleotides have shown great potential as therapeutic agents by different mechanisms [1] especially small interfering RNA duplex (siRNA) [2]. However, the drug potential of siRNAs is still limited due to low stability and inefficient delivery [3]. To increase its stability towards nuclease degradation and increase cellular uptake, modified oligonucleotides and conjugation of siRNA to different delivery carriers such as antibodies, lipids, polymers, peptides or carbohydrates have been reported [4]. Among them, lipids are being widely studied and cholesterol conjugates have been tested in vivo showing promising results [5]. Conjugation to lipophilic molecules introduces a partial hydrophobic character to the bioconjugates which may enhance their cellular uptake not only by increased membrane permeability but also through receptor mediated endocytosis [6]. Some hydrophobic modifications used on siRNA with some success are shown in Figure 1.

We recently reported the synthesis of glucose oligonucleotide conjugates where the sugar was attached to the 5'- end of the DNA strand and had different spatial presentations [7]. Cell uptake studies using flow cytometric analysis showed that conjugates with the glucose moiety linked through long spacers (15 to 18 atom distances) were better internalized than those with short linkers (4 atom distance) or than DNA control strands without sugar modification.

Then we synthesized carbohydrate-siRNA duplexes targeting tumor necrosis factor carrying one, two or four glucose and galactose residues at the $5^{\prime}$-end of the passenger strand. The modified siRNA duplexes have similar inhibitory properties than unmodified RNA duplexes in HeLa cells when transfected with oligofectamine [8]. Without transfection agent, glucosesiRNA conjugates showed no inhibition whereas galactose-siRNA duplexes on $\mathrm{HuH}-7$ cells 
which possess abundant asialoglycoprotein receptors displayed up to $25 \%$ anti-TNF inhibitory properties.

Here, the idea was to examine apolar carbohydrates linked to siRNA duplexes as new hydrophobic platforms that may improve the oligoribonucleotide cell uptake without disrupting the RNAi machinery during gene inhibition. The design consisted of per- methylated glucose attached to the 5 '-end of the passenger strand of the siRNA via two different spacers, a short ethylene glycol and a long dodecanodiol (Figure 2). A dendron scaffold was also used since it has been demonstrated that lipid-oligonucleotide conjugates presenting double-tailed lipid modifications show good results in cellular uptake [9]. A spacer-oligonucleotide conjugate was also prepared as a negative control.

Figure 1. Lipophilic vectors used in the literature to improve siRNA cell uptake. Soutschek et al. (2004) used a cholesterol, Godeau et al. (2008) used a fatty alcohol, MacKellar et al. (1992) used a double aliphatic alcohol and Nishina et al. (2008) used an alpha-tocopherol.

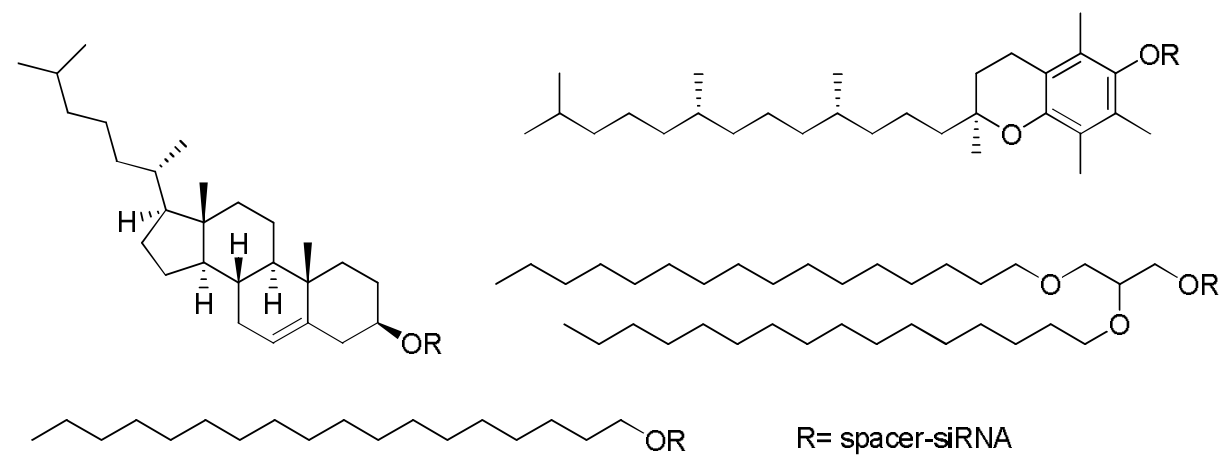

Figure 2. Structure of the carbohydrate oligoribonucleotide conjugates prepared in this study. RNA sequence is the passenger sequence: 5'-AUCUGAAGAAGGAGAAAAATT-3'. The RNA sequence of the guide is: 5'-UUUUUCUCCUUCUUCAGAUTT-3'.
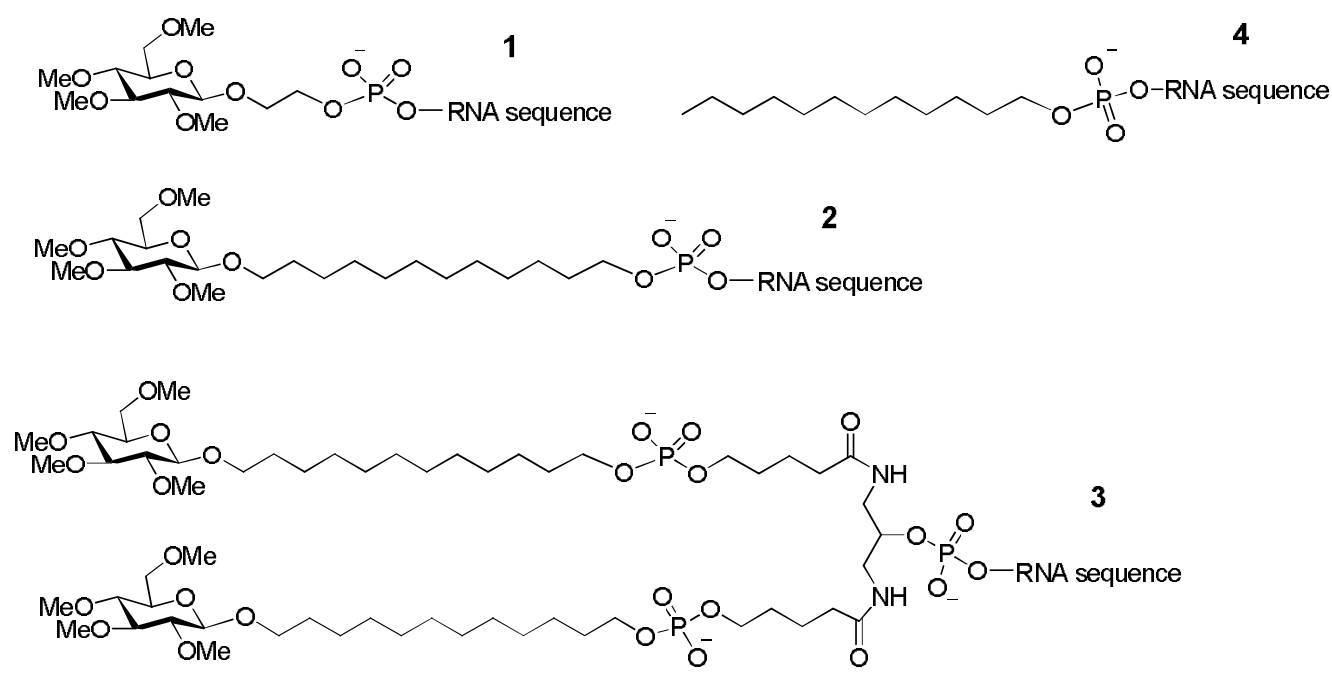
Synthesis. We synthesized carbohydrate-RNA conjugates using standard solid-phase oligonucleotide automatic synthesis using the corresponding apolar carbohydrate phosphoramidites (Scheme 1). The preparation of permethylated glucose phosphoramidite derivative 6 containing the short spacer was carried out following the same methodology described previously by our group [10]. This strategy was extended to the apolar sugar with the long dodecanodiol spacer [11]. Briefly, glycosylation of the $O$-benzyl protected dodecanodiol spacer with ?-bromoacetoglucose 7 resulted in compound 8 in $62 \%$ yield. Deprotection of the acetyl groups followed by methylation under standard conditions produced compound 9 in good overall yield (78\%, 2 steps). Then, hydrogenation and standard phosphoramidite preparation were carried out yielding permethylated glucose phosphoramidite 11 (67 and 74\%, respectively). We also prepared a permethylated cellobiose phosphoramidite 12 and a perbutylated glucose phosphoramidite 13, both with the long spacer following the same synthetic strategy. A dodecanol phosphoramidite control $\mathbf{1 4}$ was also synthesized from 1-dodecanol as described previously [11].

RNA synthesis. The siRNA sequences designed to target Renilla Luciferase gene were 5'AUCUGAAGAAGGAGAAAAATT-3' as the passenger sequence and 5'UUUUUCUCCUUCUUCAGAUTT-3' as the guide sequence. Oligoribonucleotide conjugates (1-4) were prepared using a DNA synthesizer. Apolar sugar phosphoramidites were added at the last step on the RNA synthesis. Oligonucleotides presented a major peak in HPLC chromatograms with some impurities but they were easily separated by HPLC obtaining the desired conjugates. However, isolation of siRNA conjugates containing permethylated cellobiose or perbutylated glucose, both with the long spacer, was not successful due to problems either during coupling with the corresponding phosphoramidites or during the cleavage and deprotection steps. Oligoribonucleotide sequences carrying apolar carbohydrates at the $5^{\prime}$-end of the passenger strand were hybridized to the unmodified guide strand. 

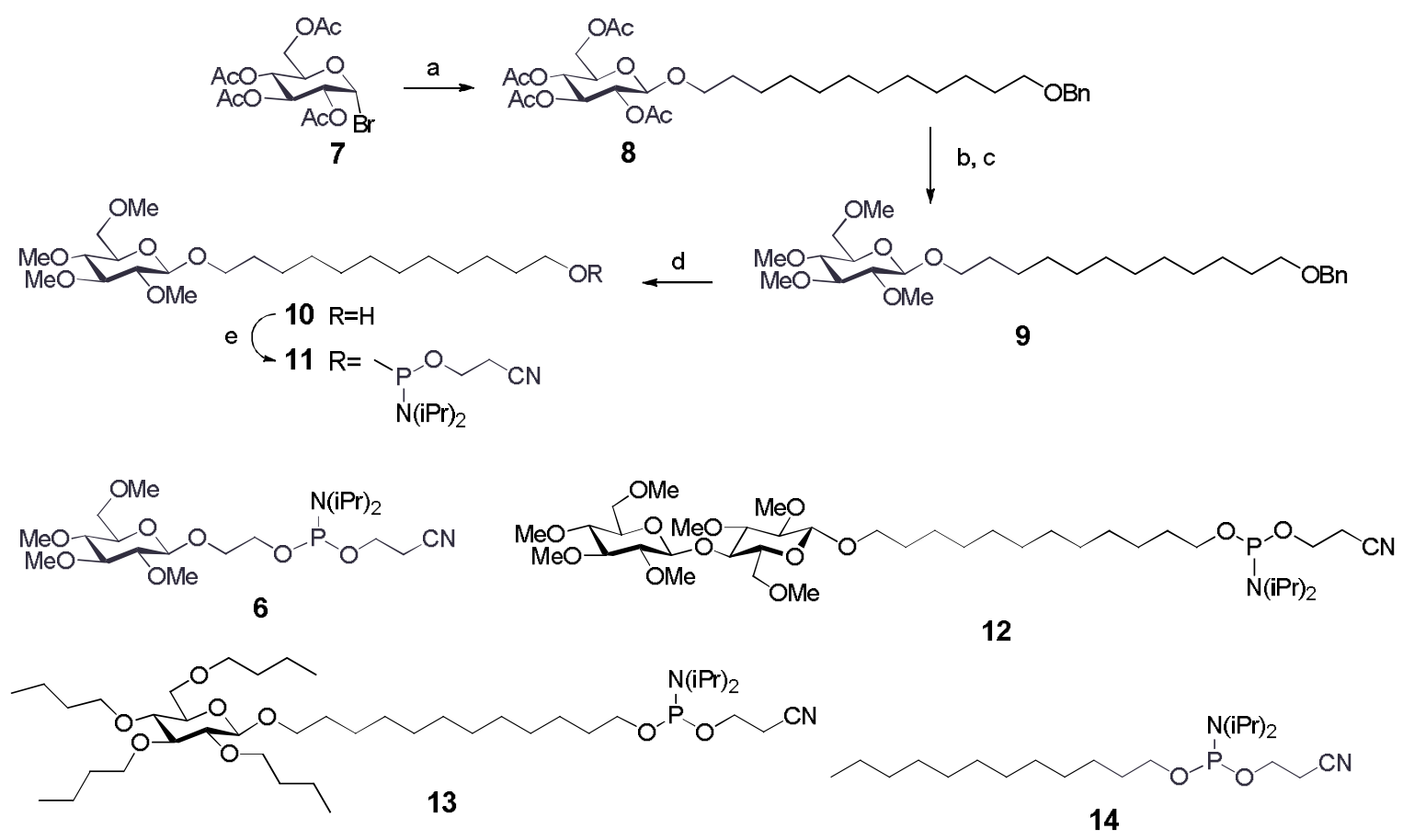

Scheme 1. Synthesis of permethylated glucose phosphoramidite derivatives 6 and 11. Compound 6 was synthesized as described in ref. 9. Reagents and conditions: a) 1,12dodecanediol, monobenzyl ether, $\mathrm{Ag}_{2} \mathrm{CO}_{3}, \mathrm{CH}_{2} \mathrm{Cl}$; b) $\mathrm{Na}_{2} \mathrm{CO}_{3}$, MeOH; c) Mel, $\mathrm{NaH}$, DMF; d) $\mathrm{H}_{2}$, $\mathrm{Pd}(\mathrm{OH})_{2}, \mathrm{THF} / \mathrm{MeOH}$; e) ) 2-cyanoethyl- $\mathrm{N}, \mathrm{N}^{\prime}$-diisopropylamino-chlorophosphoramidite, DIPEA, $\mathrm{CH}_{2} \mathrm{Cl}_{2}$.

Dual-luciferase assay. After RNA synthesis, we evaluated the effect of the above described 5'sense-carbohydrate-modified siRNAs on the ability of the corresponding conjugate derivatives to act as inhibitors of gene expression. Furthermore, we tested the ability of such conjugates to impart cell uptake. We carried out separate gene knockdown experiments in HeLa cells. In a first series of experiments, the cells were cotransfected with two luciferase plasmids (Renilla and firefly; target and internal control, respectively), with siRNA duplexes containing each of the five modified passenger strands (1-4), and with the corresponding unmodified siRNA (wt). Cotransfection of plasmids and siRNAs was carried out by using commercial cationic liposomes (Lipofectamine 2000). Twenty-two hours after transfection, the luciferase activities of the samples were measured by using a luminometer. The results, showing Renilla luciferase activity normalized to firefly luciferase, are shown in Figure 3. Interestingly, the presence of permethylated glucoseC2, and permethylated glucoseC12 at the 5 '-end of the passenger strand ( $\mathbf{1}$ and $\mathbf{2}$, respectively) did not disrupt RNAi activity. The corresponding siRNA

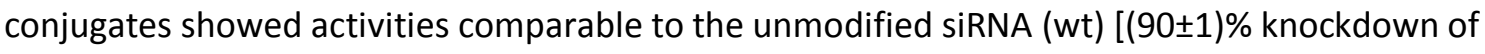
Renilla expression for unmodified wt versus $(90 \pm 1) \%$ and $(89 \pm 1) \%$ for 1 and $\mathbf{2}$, respectively]. Conjugation of permethylated glucoseC12-doubler and C12 spacer to the 5 '-end of the sense strand siRNA (conjugates $\mathbf{3}$ and $\mathbf{4}$, respectively) caused a slight decrease in activity, but gene 
silencing activity of these siRNAs was still quite remarkable: [(71 \pm 1$) \%$ and $(71 \pm 3) \%$ for $\mathbf{3}$ and $\mathbf{4}$, respectively, versus $(90 \pm 1) \%$ for $w t]$. A sequence scrambled siRNA (as negative control) was used to ensure specificity. Cells cotransfected with the scrambled siRNA showed levels of Renilla luciferase activity similar to those of cells transfected with plasmids alone. Thus, the results indicate that the siRNA 1-4 do specifically induce inhibition of expression of the target gene.

Finally, in a second series of experiments we examined the ability of conjugates 1-4 to improve cell uptake in comparison to naked siRNA (Figure 4). Three hours after transfection of the two luciferase plasmids, the cell medium was discarded and the cells were incubated with fresh medium and the siRNA derivatives in the absence of Lipofectamine 2000 (750 nM siRNA conjugates). Twenty-two hours after transfection the luciferase activities were measured as described above. Although gene knockdown was significantly less efficient than that observed for siRNA-lipofectamine complexes, results showed that siRNA conjugates $\mathbf{3}$ and $\mathbf{4}$ were able to penetrate HeLa cells in the absence of a transfection agent $[(15 \pm 3) \%$ and $(26 \pm 12) \%$ knockdown of Renilla expression for 3 and $\mathbf{4}$, respectively, versus ( $2 \pm 2) \%$ for $w t)]$.

Nuclease-resistant properties. Since the above experiments showed that most of the 5'terminal modifications could mediate RNAi activity to levels comparable to wild type siRNA, and that some siRNA conjugates were able to penetrate the cell membrane, it was of interest to study the 5'-exonuclease resistance of these 5'-modified oligonucleotides. To carry out such studies, unmodified (wt) or 5'-modified passenger strands 1-4 were incubated with Bovine Spleen Phosphodiesterase (BSP). At various time points, aliquots of the reactions were withdrawn and the samples were analyzed by polyacrylamide gel electrophoresis (Figure 5). Unmodified passenger strand showed low stability, with only $5 \%$ of the original oligonucleotide strand remaining intact after $24 \mathrm{~h}$ of incubation with BSP. Permethylated glucose C12-doublerpassenger 3 displayed a slight increase in stability, with $40 \%$ and $5 \%$ of the original oligonucleotide remaining intact through 24 and $48 \mathrm{~h}$, respectively. Very interestingly, conjugation of $\mathrm{C} 12$ to the 5 '-end of the passenger strand (5'-modified passenger strand 4 ) gave rise to a significant increase in stability, with $45 \%$ of the original strand remaining intact for 72 h. Finally, permethylated glucose C2-modified and permethylated glucose C12-modified passenger strands 1 and 2 demonstrated even higher stability, with only 20\% and 10\% of degradation after $72 \mathrm{~h}$. Further experiments should be made to understand the differences found between siRNAs.

Figure 3. Reduction in Renilla luciferase activity of modified siRNAs 1-4 in Hela cells using oligofectamine as transfecting agent for siRNA. The cells were treated with $0.8 \mathrm{nM}$ siRNA. Mean values $( \pm S D)$ from three independent experiments are presented. 


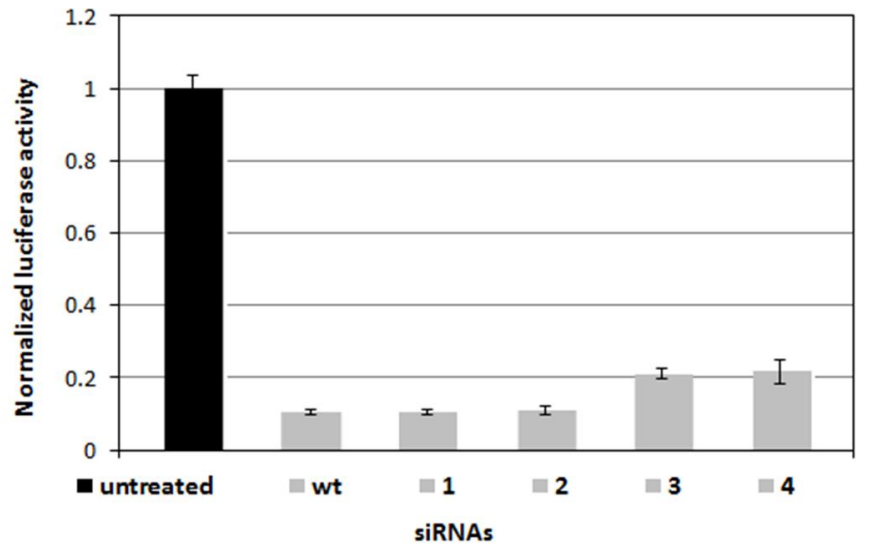

Figure 4. Reduction in Renilla luciferase activity of modified siRNAs 1-4 in Hela cells in the absence of oligofectamine as transfecting agent for siRNA. The cells were treated with $750 \mathrm{nM}$ siRNA. Mean values $( \pm S D)$ from three independent experiments are presented.

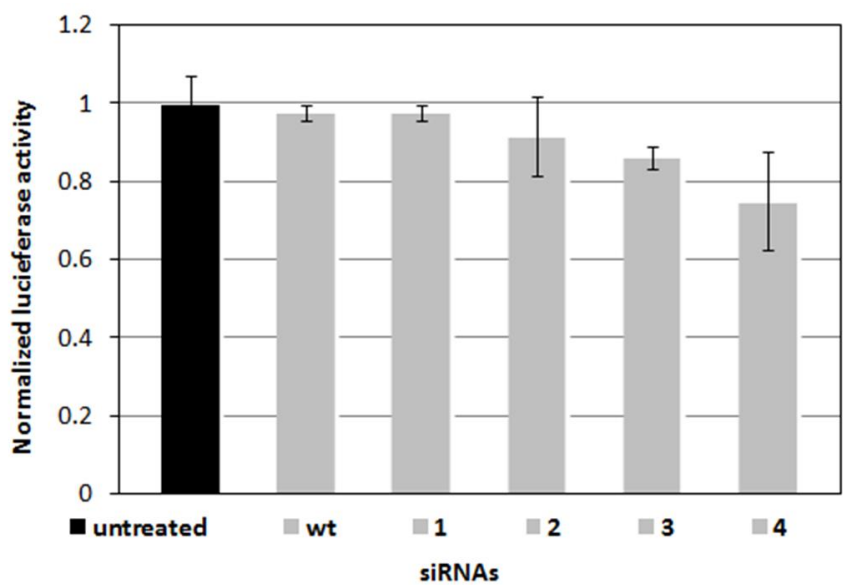

In conclusion, apolar carbohydrates covalently attached to the 5'-end of siRNA oligonucleotides have been synthesized. Three types of spacers, ethylene glycol, dodecanediol and dodecanediol symmetric doubler have been used. These modifications do not alter the RNAi machinery on HeLa cells. When siRNA modified conjugates were used without oligofectamine certain inhibition was observed. The best cellular uptake was observed for lipophilic-siRNA conjugates $\mathbf{3}$ and $\mathbf{4}$ inhibiting gene expression only up to $26 \%$ compared to the scrambled duplex. We also showed that conjugation of C12 spacer, C12-doubler, permethylated glucose $\mathrm{C} 2$ and permethylated glucose $\mathrm{C} 12$ units to the 5 '-end of the passenger strand substantially enhanced resistance to 5'-exonuclease-catalyzed hydrolysis. Altogether, our data demonstrates that apolar carbohydrates are a new tool for the development of nucleic acids-based drugs with potential value in the preparation of formulations for intravenous administration as described for lipid-modified oligonucleotides $[5,6]$. 


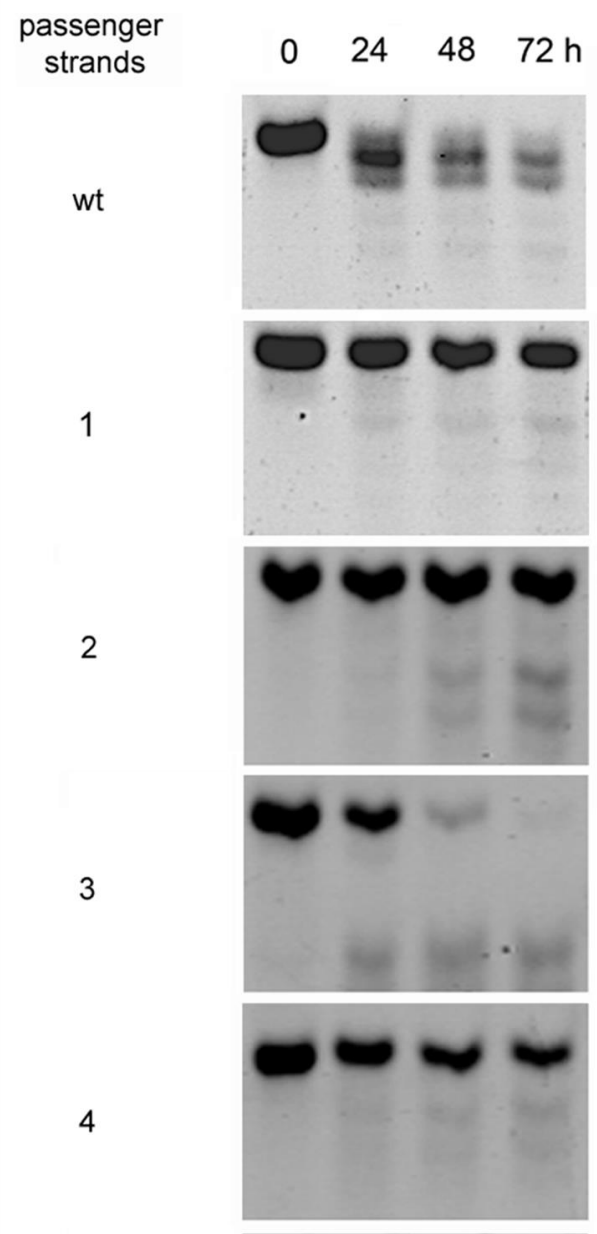

Figure 5. 20\% denaturing polyacrylamide gels depicting the time course of the BSP-catalyzed degradation of unmodified (wt) and 5'-modified passenger strands 1-4.

\section{Acknowledgments}

This study was supported by the Spanish Ministry of Economy and Competitiveness (grants CTQ2009-13705, CTQ2010-20541), Generalitat de Catalunya (2009/SGR/208). RL thanks CSIC for a JAE contract and EVC thanks Ministry of Education, Culture and Sports for a FPU grant. MT acknowledges a Juan de la Cierva contract (MICINN, Spain) for financial support.

\section{References and notes}

1. a) Opalinska, J. B.; Gewirtz, A. M. Nat Rev Drug Discov 2002, 1, 503; b) Burnett, John C.; Rossi, John J. Chem. Biol. 2012, 19, 60; c Kole, R.; Krainer, A. R.; Altman, S. Nat Rev Drug Discov 2012, 11, 125.

2. a) Castanotto, D.; Rossi, J. J. Nature 2009, 457, 426; b) Fire, A.; Xu, S.; Montgomery, M. K.; Kostas, S. A.; Driver, S. E.; Mello, C. C. Nature 1998, 391, 806.

3. a) Vaishnaw, A. K.; Gollob, J.; Gamba-Vitalo, C.; Hutabarat, R.; Sah, D.; Meyers, R.; de Fougerolles, T.; Maraganore, J. Silence 2010, 1, 14; b) Lares, M. R.; Rossi, J. J.; Ouellet, D. L. Trends in Biotechnol. 2010, 28, 570.

4. a) De Paula, D.; Bentley, M. V. L. B.; Mahato, R. I. RNA 2007, 13, 431; b) Jeong, J. H.; Mok, H.; Oh, Y. K.; Park, T. G. Bioconjugate Chem. 2009, 20, 5; c) Peer, D.; Lieberman, J. Gene Ther 2011, 18, 1127. 
5. a) Soutschek, J. et al. Nature $2004,432,173$; b) Zimmermann, T. S. et al Nature 2006, 441, 111.

6. a) MacKellar, C.; Graham, D.; Will, D. W.; Burgess, S.; Brown, T. Nucleic Acids Res 1992, 20, 3411; b) Godeau, G.; Staedel, C.; Barthelemy, P. J Med Chem 2008, 51, 4374; c) Nishina, K.; Unno, T.; Uno, Y.; Kubodera, T.; Kanouchi, T.; Mizusawa, H.; Yokota, T. Mol. Ther. 2008, 16, 734; d) Grijalvo, S.; Ocampo, S. M.; Perales, J. C.; Eritja, R. Chem Biodiversity 2011, 8, 287; e) Petrova, N. S. et al Nucleic Acids Res 2012, 40, 2330.

7. Ugarte-Uribe, B.; Pérez-Rentero, S.; Lucas, R.; Aviñó, A.; Reina, J. J.; Alkorta, I.; Eritja, R.; Morales, J. C. Bioconjugate Chem. 2010, 21, 1280.

8. Aviñó, A.; Ocampo, S. M.; Lucas, R.; Reina, J. J.; Morales, J. C.; Perales, J. C.; Eritja, R. Mol Divers 2011, 15, 751.

9. a) MacKellar, C., Graham, D., Will, D. W., Burgess, S., and Brown, T. Nucleic Acids Res. 1992, 20, 3411; b) PhD Thesis Begoña Ugarte-Uribe, 2012, UPV/EHU.

10. Lucas, R.; Vengut-Climent, E.; Gómez-Pinto, I.; Avinnó, A.; Eritja, R.; González, C.; Morales, J. C. Chem Commun 2012, 48, 2991.

11. Laing, B. M.; Barrow-Laing, L.; Harrington, M.; Long, E. C.; Bergstrom, D. E. Bioconjugate Chem. 2010, 21, 1537. 